\title{
The Nature of Quantum States Created by One Photon Absorption: Pulsed Coherent vs. Pulsed Incoherent Light
}

\author{
Alex C. Han ${ }^{1}$, Moshe Shapiro ${ }^{1,2}$ and Paul Brumer ${ }^{3}$ \\ ${ }^{1}$ Department of Physics and Astronomy, and ${ }^{2}$ Department of Chemistry, \\ The University of British Columbia, Vancouver, and ${ }^{3}$ Chemical Physics Theory Group, \\ Department of Chemistry, The University of Toronto, Toronto, Canada
}

\begin{abstract}
We analyze electronically excited nuclear wave functions and their coherence when subjecting a molecule to the action of natural, pulsed incoherent solar-like light, and to that of ultrashort coherent light assumed to have the same center frequencies and spectral bandwidths. Specifically, we compute the spatio-temporal dependence of the excited wave packets and their electronic coherence for these two types of light sources, on different electronic potential energy surfaces. The resultant excited state wave functions are shown to be qualitatively different, reflecting the light source from which they originated. In addition, electronic coherence is found to decay significantly faster for incoherent light than for coherent ultrafast excitation, for both continuum and bound wave packets. These results confirm that the dynamics observed in studies using ultrashort coherent pulses are not relevant to naturally occurring solar-induced processes such as photosynthesis and vision.
\end{abstract}

\section{Introduction}

An increasing number of studies in recent years have identified surprisingly long-lived coherences in biological systems, especially in photosynthesic light harvesting molecules [1] []. Such long-lived coherences were detected in studies such as two-colour photon echo spectroscopy 2. 4, angle-resolved coherent optical wave-mixing [3, and phase-stabilized 2D electronic spectroscopy [7]. Following these findings, it has been suggested that quantum coherence may play an important role in biological processes, for example in the proton and energy transfer that follow the photo-excitation step. A challenging question is to what extent the dynamics observed in such experiments, which utilize coherent laser light sources, are relevant for processes induced by incoherent sources such as sunlight, even when the two types of light share the same center frequencies and spectral bandwidths.

Several previous studies have analyzed various effects of incoherent excitation sources on the resultant molecular dynamics. These include an analysis of the excited state survival probability as a function of the incoherence of the light 8 , effects of incoherent fields on the photoisomerization yields [9], a quantum-optical formulation of the state of the molecule after photon-absorption [10], studies of open system dynamics relevant to photosynthetic complexes [11, and general features of the response of open systems to incoherent excitation 12 . However, none of these studies looked directly at the spatial shape of the wave packets and the associated temporal evolution and coherence properties resulting from excitation with incoherent vs. coherent light sources. Such studies are particularly important in comparing the nature of the excited quantum state prepared by these light sources.

In this paper we concentrate on the actual spatiotemporal shape of the wave functions resulting from photo-excitation, a primary entity that effectively brings out the differences between the two types of light-induced excitations mentioned above. In addition, we consider the photo-excitation of a model molecule into a superposition of two electronic states, and contrast the calculated coherences induced by incoherent solar-like light with the outcome of excitation by an ultrashort coherent pulse with the same center frequency and spectral bandwidth.

Our main theoretical tool is a time-dependent quantum-theoretic calculation of the different nuclear wave packets resulting from the photo-excitation step. Optical incoherence is simulated by introducing random jumps in the phases and center frequencies of the excitation pulse, with the degree of optical incoherence being controlled by the frequency and amplitudes of these random jumps. Both the spatial structure and time dependence of the resultant wave packet dynamics is then analyzed for a variety of potential energy surfaces (PES).

\section{Theory of "random" and coherent wave packet dynamics}

Consider first the case in which a nuclear wave packet is photo-excited to a single excited PES. The molecule which is assumed to be initially in the ground electronic state is excited by a light source, treated as a classical time-varying electric field, to a dissociative or bound excited state. The time-evolution of the total molecular quantum state can be described by a (stochastic) Schrödinger's equation, written in the adiabatic (Born Oppenheimer) electronic basis as

$$
i \hbar \frac{d}{d t} \underline{\Psi}(\mathbf{R}, t)=\underline{\underline{H}}(t) \underline{\Psi}(\mathbf{R}, t),
$$

where

$$
\underline{\Psi}(\mathbf{R}, t) \equiv\left(\begin{array}{c}
\Psi_{g}(\mathbf{R}, t) \\
\Psi_{x}(\mathbf{R}, t)
\end{array}\right)
$$


with $\Psi_{g}\left(\Psi_{x}\right)$ being the nuclear wave packet in the ground (excited) electronic manifold. The total Hamiltonian matrix consists of a molecular part and an electric-dipole Matter-Radiation interaction part

$$
\begin{aligned}
& \underline{\underline{\mathrm{H}}}(t)=\underline{\underline{\mathrm{H}}}^{M}+\underline{\underline{\mathrm{H}}}^{M R}(t), \\
& \underline{\underline{\mathrm{H}}}^{M}=\left(\begin{array}{cc}
H_{g} & 0 \\
0 & H_{x}
\end{array}\right)=\left(\begin{array}{cc}
T_{N}+W_{g} & 0 \\
0 & T_{N}+W_{x}
\end{array}\right), \\
& \underline{\underline{\mathrm{H}}}^{M R}(t)=\left(\begin{array}{cc}
0 & V^{* M R}(t) \\
V^{M R}(t) & 0
\end{array}\right) .
\end{aligned}
$$

The nuclear kinetic energy operator $T_{N}$ and the ground and excited PES functions $W_{g, x}$ of the above depend on the set of nuclear coordinates $\mathbf{R}$. The Matter-Radiation interaction term is given as,

$V^{M R}(\mathbf{R}, t)=-\left[\int \psi_{x}^{e *}\left(\mathbf{R}, q_{e}\right) \mathbf{d}\left(\mathbf{R}, q_{e}\right) \psi_{g}^{e}\left(\mathbf{R}, q_{e}\right) d q_{e}\right] \cdot \hat{\epsilon} E(t)$,

where $q_{e}$ is the set of electronic coordinates, $E(t) \equiv$ $\mathcal{E}_{L}(t) \cos (\omega t+\Phi)$ is the electric field, $\mathbf{d}\left(\mathbf{R}, q_{e}\right)$ is the dipole moment, and $\hat{\epsilon}$ is the unit polarization vector.

It is most convenient to solve the Schrödinger equation in the interaction representation (with the $\mathbf{R}$ variable temporarily suppressed for brevity)

$i \hbar \frac{d}{d t}\left[\underline{\underline{\mathrm{U}}}^{\dagger}\left(t-t_{0}\right) \cdot \underline{\Psi}\left(t_{0}\right)\right]=\underline{\underline{\mathrm{H}}}_{I}^{M R}(t) \cdot\left[\underline{\underline{\mathrm{U}}}^{\dagger}\left(t-t_{0}\right) \cdot \underline{\Psi}\left(t_{0}\right)\right]$,

where $t_{0}$ represents some initial time long before the onset of $\underline{\mathrm{H}}^{M R}(t)$, and

$\underline{\underline{\mathrm{H}}}_{I}^{M R}(t) \equiv \underline{\underline{\mathrm{U}}}^{\dagger}\left(t-t_{0}\right) \underline{\underline{\mathrm{H}}}^{M R}(t) \underline{\underline{\mathrm{U}}}\left(t-t_{0}\right)$,

with the (interaction representation) evolution operators defined as

$\underline{\underline{\mathrm{U}}}\left(t_{f}-t_{i}\right) \equiv e^{-\frac{i}{\hbar} \underline{\underline{\underline{H}}}^{M}\left(t_{f}-t_{i}\right)}=\left(\begin{array}{cc}U_{g}\left(t_{f}-t_{i}\right) & 0 \\ 0 & U_{x}\left(t_{f}-t_{i}\right)\end{array}\right)$.

Assuming the laser field intensity is weak enough so that first-order perturbation theory is valid, the state in the interaction representation assumes the form

$$
\underline{\underline{U}}^{\dagger}\left(t-t_{0}\right) \cdot \underline{\Psi}(t)=\underline{\Psi}\left(t_{0}\right)+\frac{1}{i \hbar} \int_{t_{0}}^{t} \underline{\underline{H}}_{I}^{M R}\left(t^{\prime}\right) \cdot \underline{\Psi}\left(t_{0}\right) d t^{\prime},
$$

or in the Schrödinger's representation,

$$
\underline{\Psi}(t)=\underline{\underline{\mathrm{U}}}\left(t-t_{0}\right) \cdot\left[1+\frac{1}{i \hbar} \int_{t_{0}}^{t} \underline{\underline{H}}_{I}^{M R}(t) d t^{\prime}\right] \cdot \underline{\Psi}\left(t_{0}\right)
$$

In order to gain physical insight we now consider excitation by a $E(\tau) \delta(t-\tau)$ pulse, for which $V^{M R}(\mathbf{R}, t)=$ $D(\mathbf{R}) E(\tau) \delta(t-\tau)$, where

$$
D(\mathbf{R}) \equiv-\hat{\epsilon} \cdot \int \psi_{x}^{e *}\left(\mathbf{R}, q_{e}\right) \mathbf{d}\left(\mathbf{R}, q_{e}\right) \psi_{g}^{e}\left(\mathbf{R}, q_{e}\right) d q_{e}
$$

The assumption that the molecule is initially in the ground electronic manifold, $\underline{\Psi}\left(\mathbf{R}, t_{0}\right)=\left(\begin{array}{c}\Psi_{g}\left(\mathbf{R}, t_{0}\right) \\ 0\end{array}\right)$, reduces Eq. (7) to

$\Psi_{x}(\mathbf{R}, t)=\frac{h(t-\tau)}{i \hbar} U_{x}(t-\tau) D(\mathbf{R}) E(\tau) U_{g}\left(\tau-t_{0}\right) \Psi_{g}\left(\mathbf{R}, t_{0}\right)$,

where

$$
h(t-\tau)=\left\{\begin{array}{l}
1 \text { for } t>\tau \\
\frac{1}{2} \text { for } t=\tau \\
0 \text { for } t<\tau
\end{array}\right.
$$

is the Heaviside function. The physical picture that emerges is that no population is excited before the arrival of the delta pulse, and that exactly at $t=\tau$, where $h(0)=1 / 2$, and $U_{e}(\tau-\tau)=1$, we have that

$$
\begin{aligned}
\Psi_{x}(\mathbf{R}, \tau) & =\frac{D(\mathbf{R})}{2 i \hbar} E(\tau) U_{g}\left(\tau-t_{0}\right) \Psi_{g}\left(\mathbf{R}, t_{0}\right) \\
& =\frac{D(\mathbf{R})}{2 i \hbar} E(\tau) \Psi_{g}(\mathbf{R}, \tau) .
\end{aligned}
$$

Thus, at $t=\tau$ we form on the excited PES a "replica" of the ground state wave packet times $D(\mathbf{R}) E(\tau) / 2 i \hbar$, that evolves at $t>\tau$ as

$$
\Psi_{x}(\mathbf{R}, t>\tau)=U_{x}(t-\tau) \Psi_{x}(\mathbf{R}, \tau)
$$

Using the above insight we now expand any timedependent function for the electric field as a series of delta pulses,

$$
E(t)=\int_{-\infty}^{\infty} E(\tau) \delta(t-\tau) d \tau
$$

and generalize Eq. (8) for a general pulse to obtain that

$$
\Psi_{x}(\mathbf{R}, t)=\int_{-\infty}^{t} \frac{U_{x}(t-\tau)}{i \hbar} D(\mathbf{R}) E(\tau) U_{g}\left(\tau-t_{0}\right) \Psi_{g}\left(\mathbf{R}, t_{0}\right) d \tau
$$

The excited wave packet is seen to be a sum of replicas launched by all the delta pulses up till time $t$, evolving according to the excited state Hamiltonian $H_{x}$ [14, 15]. Written in differential form

$i \hbar \frac{d}{d t} \Psi_{x}(\mathbf{R}, t)=H_{x} \Psi_{x}(\mathbf{R}, t)+D(\mathbf{R}) E(t) U_{g}\left(t-t_{0}\right) \Psi_{g}\left(\mathbf{R}, t_{0}\right)$,

which is an inhomogeneous Schrödinger's equation for the excited state nuclear wave function $\Psi_{x}(\mathbf{R}, t)$ whose source term is the field times the dipole operator times the ground electronic state.

The great advantage of Eq. (14) is that it allows us to easily realize varying degrees of incoherences in the light sources by introducing random jumps in the center frequency and the carrier-envelope phase of a select 

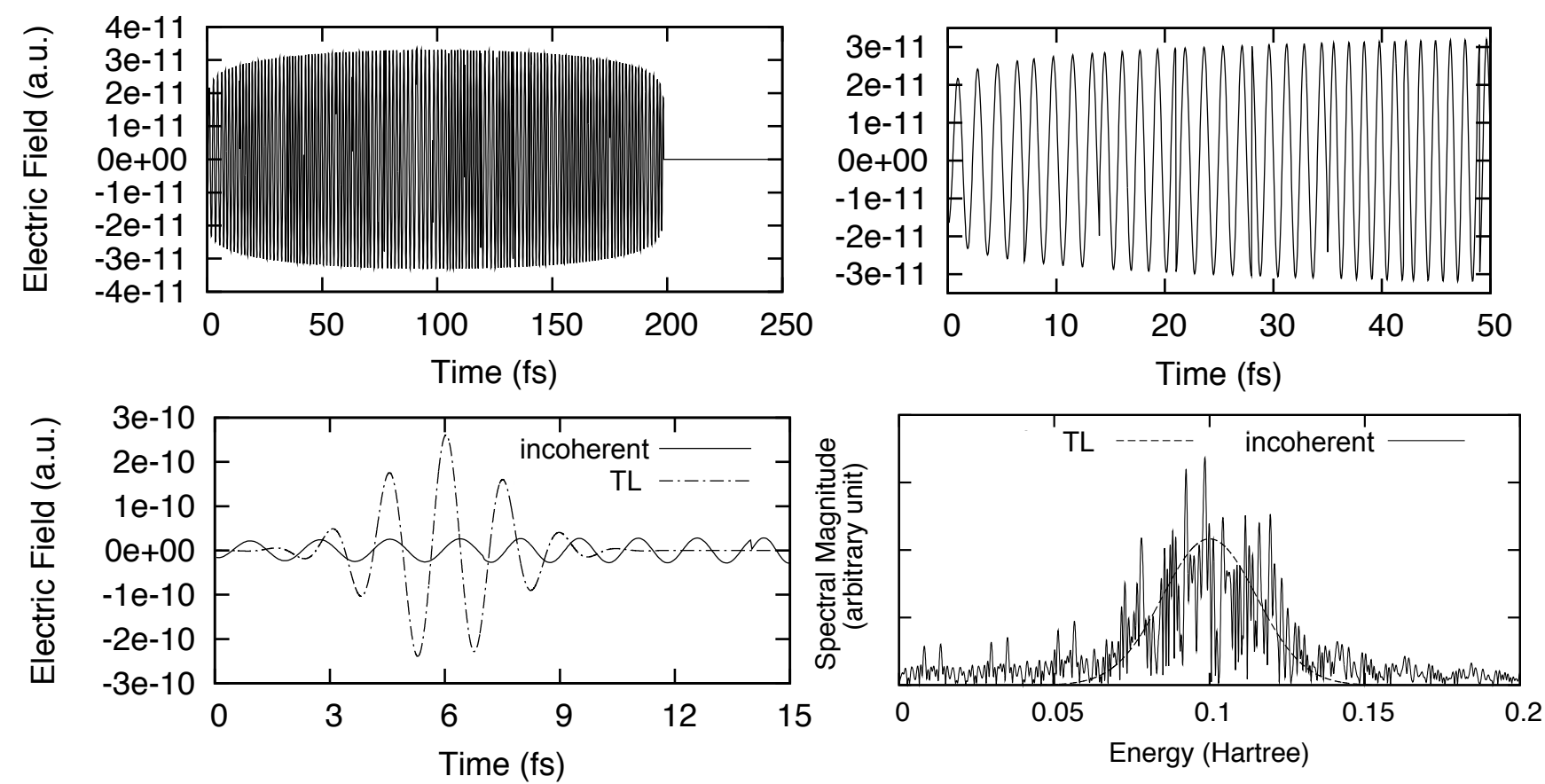

FIG. 1: Upper left panel - a sample realization of incoherent light. Upper right panel - magnified view. The center wavelength is $\sim 455 \mathrm{~nm}$ (corresponding to center energy of 0.1 Hartree). The pulse has envelope function $\mathcal{E}_{L}(t) \sim[\sin (\pi t / 200 \mathrm{fs})]^{0.1}$ for $0<t<200 \mathrm{fs}$, and is zero elsewhere. The incoherent light simulates solar radiation by introducing, every 7 fs on the average, a random phase jump in the $[-\pi, \pi]$ range, and a center frequency shift in the \pm 0.0175 Hartrees range. Lower left panel - A coherent pulse whose envelope is $\mathcal{E}_{L}(t) \sim \exp \left[-(t-6 \mathrm{fs})^{2} / 2(1.6 \mathrm{fs})^{2}\right]$. Lower right panel - The frequency profile of the pulses of coherent and incoherent light, demonstrating that both light sources share the same center frequency and the same spectral bandwidth.

number of the $\delta$ function components of the electric field. Specifically, we write the magnitude of each $\delta$ function component of Eq. 12 as

$$
E(\tau)=\mathcal{E}_{L}(\tau) \cos \left\{\left[\omega_{L}+\Delta \omega(\tau)\right] \tau+\Phi(\tau)\right\} .
$$

The real-valued functions $\Delta \omega(\tau)$ and $\Phi(\tau)$, which are taken to be constant (zero) in time for coherent fields, serve as random variables for incoherent light.

\section{Results and Discussion}

Consider then the results of numerical simulations by pulsed coherent and incoherent light. Figure 1 displays the coherent pulse, and one specific realization of the incoherent light pulse, in which we have chosen similar average values for the center frequency and bandwidth, with rate of jumps of the center frequency shifts and phase interruptions modelling typical solar values. The total energy flux, $\int_{-\infty}^{\infty}|E(t)|^{2} d t$, is kept the same for the two types of pulses.

As a model molecular system we consider the PES of the $\mathrm{H}_{2}^{+}$molecular ion. We examined the photo-excitation from the ground $1 \sigma_{g}$ electronic state, to two (dissociative or bound) excited states of the $1 \sigma_{u}$ symmetry. We assumed that these two states are decoupled from one another in order to reduce the dynamics to that of a sum of the individual ground to excited state transitions of Eq. 14. Although this is a model system, the general results that emerge are expected to apply to a wide variety of systems.

We assume that at $t_{0}=0$ the ground state wave function is a ro-vibrational energy eigenstate with zero angular momentum. The transition dipole function for a linearly polarized excitation field along the $z$-axis, is given as, $D(\mathbf{R})=D(R) Y_{1}^{0}(\theta)$ where $R$ is the internuclear separation. We model $|D(R)| \approx R / 2$ a.u. as in $\mathrm{H}_{2}^{+}$[13.

Writing the $J=1$ wave function as a product of vibrational and angular parts $\Psi_{x}(\mathbf{R}, t)=Y_{1}^{0}(\theta, \phi) \psi_{x}(R, t)$, and using atomic units, transforms Eq. (13) to

$\psi_{x}(R, t)=\int_{0}^{t} U_{x}^{J=1}(t-\tau, R) D(R) E(\tau) e^{-i E_{g} \tau} \psi_{g}(R, 0) d \tau$.

The excited state propagator for the $J=1$ channel is given as

$$
U_{x}^{J=1}(t-\tau, R)=\exp \left[-i H_{x}^{J=1}(R)(t-\tau)\right],
$$


with TL pulse

excited electronic state-1
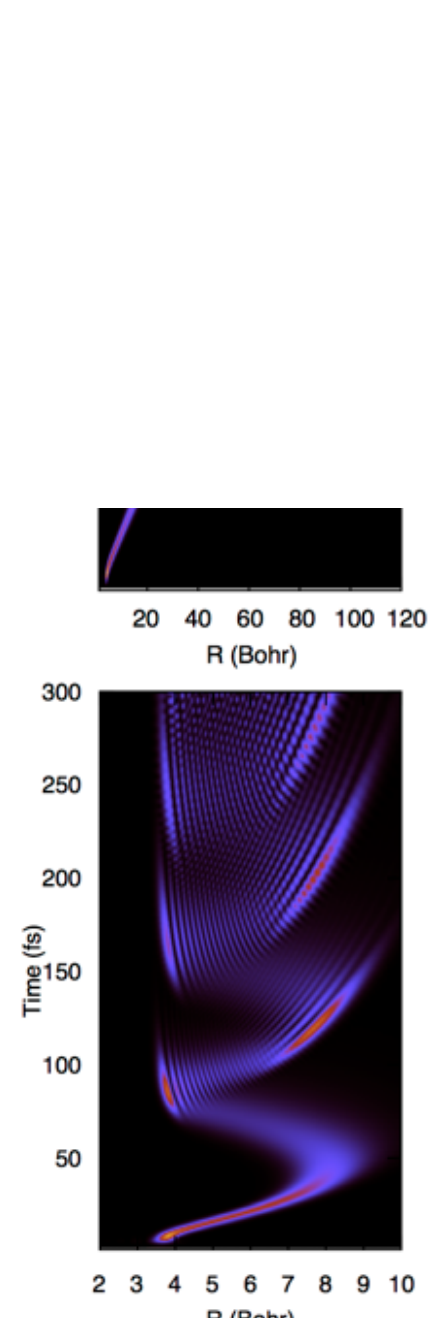

R (Bohr) excited electronic

state-2

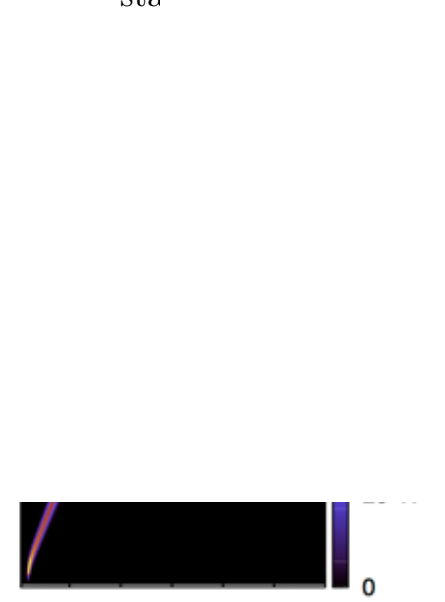

$\begin{array}{llllll}20 & 40 & 60 & 80 & 100 & 120\end{array}$ R (Bohr)

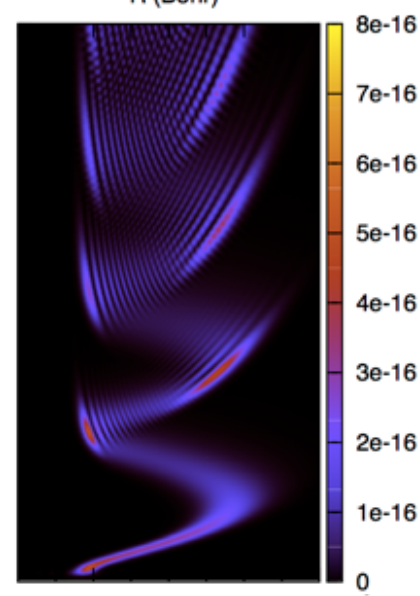

$\begin{array}{lllllllll}2 & 3 & 4 & 5 & 6 & 7 & 8 & 9 & 10\end{array}$ R (Bohr) with incoherent pulse excited electronic

excited electronic

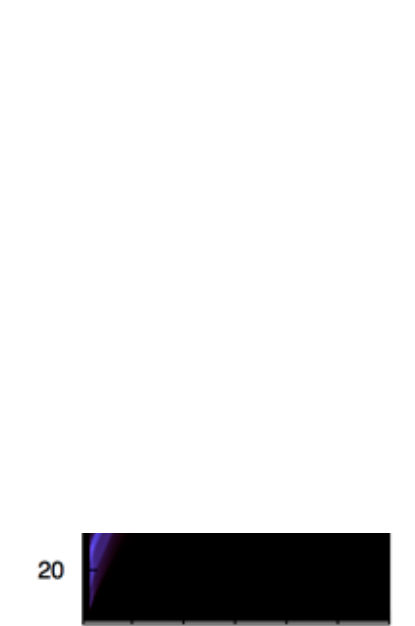

$\begin{array}{llllll}20 & 40 & 60 & 80 & 100 & 120\end{array}$ R (Bohr)

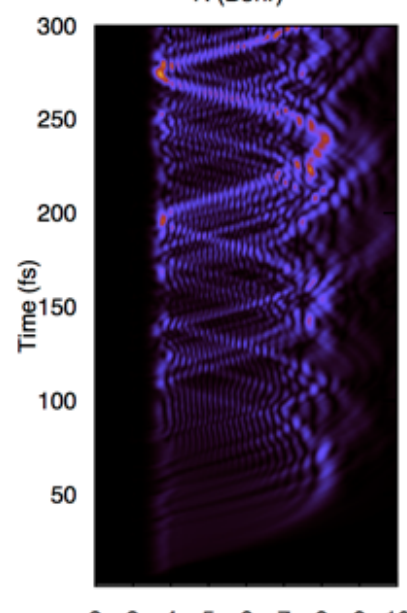

$\begin{array}{lllllllll}2 & 3 & 4 & 5 & 6 & 7 & 8 & 9 & 10\end{array}$ R (Bohr) state-2

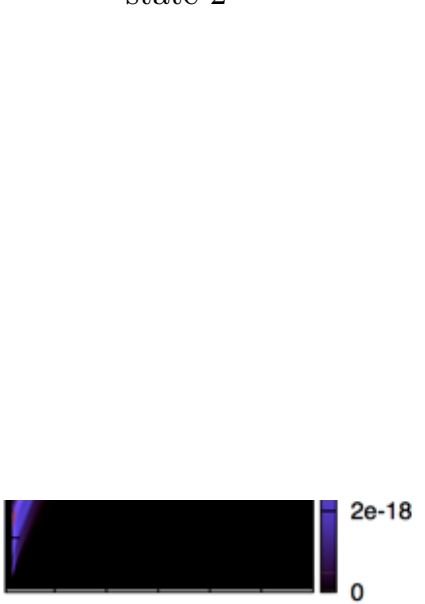

$\begin{array}{llllll}20 & 40 & 60 & 80 & 100 & 120\end{array}$

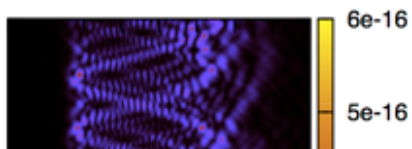

$4 e-16$

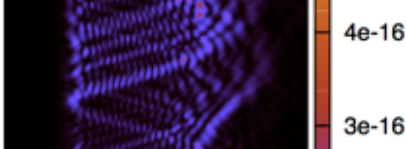

$3 e-16$

$2 e-16$

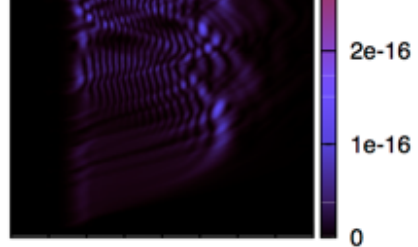

$\begin{array}{lllllllll}2 & 3 & 4 & 5 & 6 & 7 & 8 & 9 & 10\end{array}$

R (Bohr)

FIG. 2: The spatio-temporal plots of the modulus-squared radial wave functions of the vibrational wave packets, $u_{x}^{(1,2)}(R, t)$. Top row is for purely repulsive excited state- 1 and state- 2 potentials, and bottom row is for bound Morse wells. The functional forms for both types of PES correspond to the first and third situation in Figure 3 .

where

$$
\begin{aligned}
H_{g, x}^{J}(R) & =\frac{-1}{2 \mu}\left[\frac{d^{2}}{d R^{2}}+\frac{2}{R} \frac{d}{d R}\right]+V_{g, x}^{J}(R) \\
V_{g, x}^{J}(R) & =\frac{J(J+1)}{2 \mu R^{2}}+W_{g, x}(R),
\end{aligned}
$$

with $\mu$ being the reduced mass associated with the $R$ coordinate.

Using Eq. (14) and Eq. (16), we have

$\frac{d \psi_{x}(R, t)}{d t}=-i H_{x}^{J=1}(R) \psi_{x}(R, t)+D(R) E(t) e^{-i E_{g} t} \psi_{g}(R)$.

Finally transforming to radial wave functions $u_{g, x}(R, t)=R \psi_{g, x}(R, t)$, we obtain the radial ana- $\log$ of the inhomogeneous Schrödinger equation,

$$
\begin{aligned}
\frac{d}{d t} u_{x}(R, t) & =-i\left[\frac{-1}{2 \mu} \frac{d^{2}}{d R^{2}}+V_{x}^{J=1}(R)\right] u_{x}(R, t) \\
& +D(R) E(t) e^{-i E_{g} t} u_{g}(R) .
\end{aligned}
$$

Modelling the electric field $E(t)$ as in Fig. 1 and choosing $u_{g}(R)$ as the $v=5$ vibrational state, we used Eq. (20) to propagate the pair of excited radial wave functions $u_{x}^{(1)}(R, t)$ and $u_{x}^{(2)}(R, t)$ from $t=0$ to $300 \mathrm{fs}$, using time-steps of $0.003 \mathrm{fs}$ and radial-steps of 0.02 Bohrs. We analyze the motion of the $u_{x}^{(1)}(R, t)$ and $u_{x}^{(2)}(R, t)$ pair that has originated from the same ground electronic state for two cases: 1) Continuum dynamics for a pair of purely repulsive exponentially-decaying excited potentials. 2) Bound state dynamics for a pair of Morse po- 
tentials, where a heavier reduced mass, $\mu=5 \times 918$ a.u. is used. Sample plots of the wave packets, for these two cases, subject to the coherent and incoherent modes of excitation, are presented in Figs. 2 In the case of the incoherent excitation, the results of a single random realization is shown.

The completed wave packets resulting from incoherent light excitation are seen to be choppy and highly unstructured. In the case of the repulsive potentials, following the excitation step, these wave packets spread out in configuration space much more rapidly, while covering much larger extensions, than do the coherently excited cases. The situation is even more dramatically seen in the bound state excitation cases where the wave packets resulting from the incoherent light excitation exhibit a significantly smaller number of coherent oscillations as compared to excitation by coherent pulses, and a highly irregularly structured wave function is seen.

The above results show characteristics of the excited state wave functions on individual electronic states. Also of interest, in particular with respect to experimental studies of long-lived coherences, is the persistence of electronic coherence, a property of several electronic levels. To examine this we calculate the electronic coherence given by $\rho_{1,2}(t)$, the off-diagonal element of the density matrix of the two electronic states, traced over the nuclear spatial coordinate,

$$
\rho_{1,2}(t)=\int u_{x}^{*(1)}(R, t) u_{x}^{(2)}(R, t) d R .
$$

Because the coherence is obtained via first-order perturbation theory, it is convenient to normalize $\rho_{1,2}(t)$ by dividing it by $\rho_{1,1}(t)+\rho_{2,2}(t)$, the density matrix trace, giving

$$
C(t)=\frac{\rho_{1,2}(t)}{\rho_{1,1}(t)+\rho_{2,2}(t)} .
$$

In this way we factor out the effects of the total excitation yield that are of no interest for this comparative study. Note also that Eq. (21), by averaging over vibrations, includes the effect of decoherence, due to the vibrational degree of freedom, on the electronic coherences. The resultant normalized coherences $C(t)$ are plotted in Fig. 3. for different choices of the PES for the two electronic states, under the action of the two types of fields.

These plots clearly show that for incoherent field excitations the magnitude of the coherence $C(t)$ decays at a much faster rate than that of the coherent pulse, approaching a much lower asymptotic value than that of the coherent case. This is especially so, as shown in the middle row of Fig. 3, when coherence $C(t)$ is not significant, to begin with, in the coherent case, due to small Franck-Condon overlap regions of the two surfaces. In the case of the bound Morse potential wells, clear and gradual decoherence of the two wave packets is observed for the case of coherent excitation. This is in strong contrast with the incoherent pulse excitation, where the coherence $C(t)$ decays almost immediately.

In either of the three cases the final electronic coherence induced by the incoherent field is a small fraction of the coherence generated by the coherent case. Thus we find that the incoherence of the light eliminates "long lived coherences" when the excitation is carried out with pulses of solar-like incoherent sources.

Several comments are in order. First, note that decoherence observed here does not include additional environmental effects, since the molecule is isolated, i.e. the system is closed. Rather, the observed fast coherence loss arises, in the incoherent light case, from the nature of the two wave packets as they move away from one another. That is, the molecular vibration serves as the decohering environment 16, and it is particularly effective in this case due to the highly unstructured and choppy nuclear wave functions created by incoherent light. If, in addition, coupling to an external environment is present, the decay of electronic coherence will also reflect this coupling [12. As a consequence, long lived coherences are not expected to persist in realistic open systems irradiated with incoherent sources.

Second, note that the incoherent light source used above acts over a $200 \mathrm{fs}$ time scale. Thus, although the $7 \mathrm{fs}$ coherence time of solar radiation is represented in this computation, there are two significant differences between the results of this computation, and that which would result from solar irradiation, which acts over far longer times, and that is is effectively CW. First, any pulse possesses some degree of coherence due to the pulse envelope [8. Hence, the "incoherent pulse" used here possesses more coherence than would natural solar radiation that is incident for minutes or longer. Second, at such long times, as discussed elsewhere [8, 10, long time excitation of isolated molecules using natural incoherent light leads, when averaged over realizations, to stationary eigenstates of the Hamiltonian that do not evolve in time. Relaxation in open systems also leads to mixtures of stationary states [12].

\section{Conclusions}

This paper has shown that the nature of the lightinduced wave function, as well as the rates of electronic coherence in molecules, strongly reflect the coherence properties of the incident radiation. Coherent light pulses produce well localized wave packets whereas incoherent pulses produce irregularly structured coordinate space densities. In addition, we have calculated electronic coherences in the photo-excitation of a molecule into a superposition of two electronic states, using the two types of electromagnetic pulses with different degrees of coherence. The results show decreased electronic coherence 

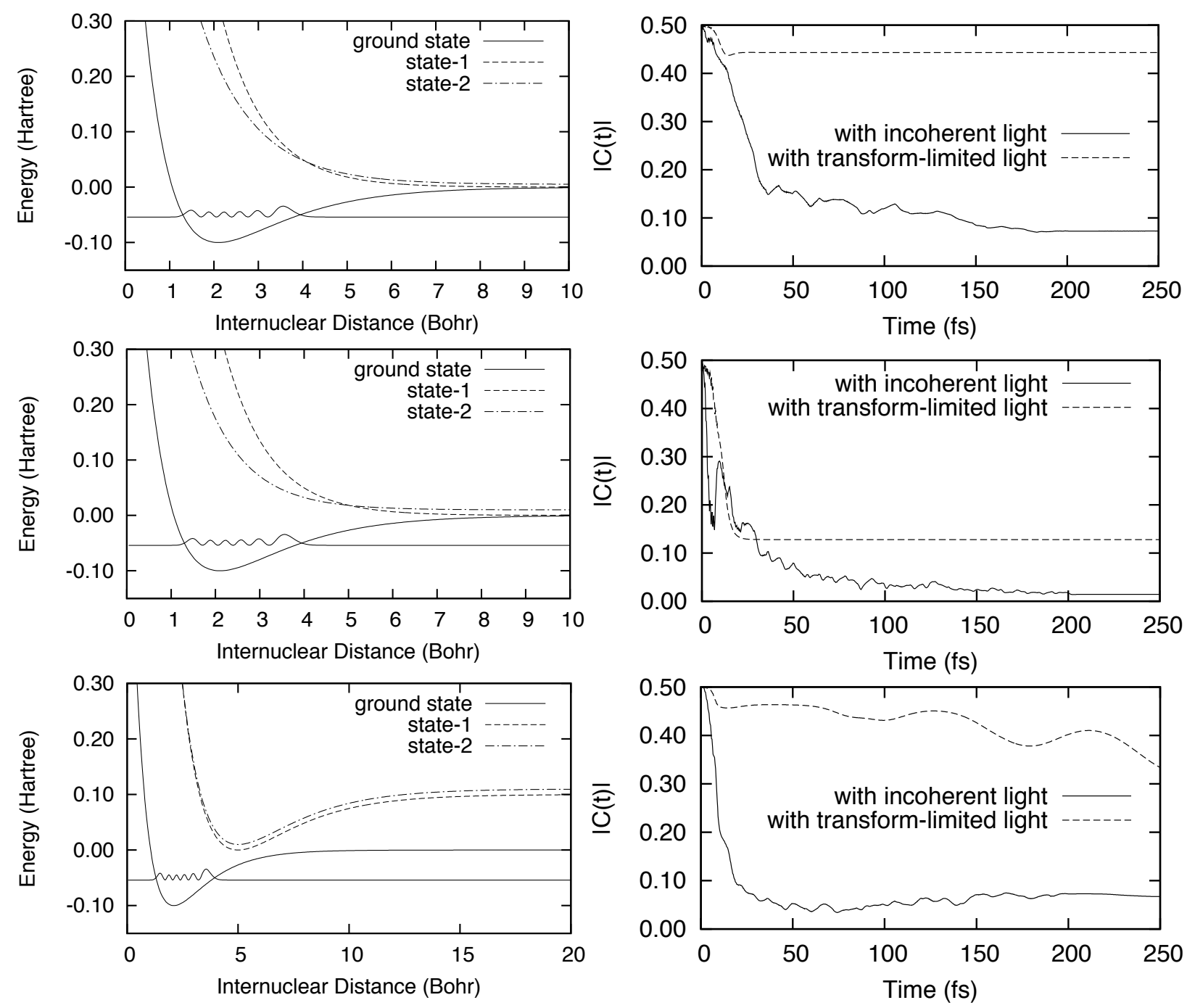

FIG. 3: Left column - PES and the $v=5$ vibrational wave function. Top panel Repulsive PES of the form, $W_{x}^{(i)}(R)=$ $W_{0}^{(i)} \exp \left[-\left(R-R_{0}^{(i)}\right) / a^{(i)}\right]+W_{\infty}^{(i)}, i=1,2$; where $W_{0}^{(1)}=0.1$ Hartree, $R_{0}^{(1)}=3.3 \mathrm{Bohr}, a^{(1)}=1.0 \mathrm{Bohr}, W_{\infty}^{(1)}=0 ; W_{0}^{(2)}=0.1$ Hartree, $R_{0}^{(2)}=3.0 \mathrm{Bohr}, a^{(2)}=1.2 \mathrm{Bohr}, W_{\infty}^{(2)}=0.005$ Hartree. Middle panel: $W_{0}^{(1)}=0.1$ Hartree, $R_{0}^{(1)}=3.3 \mathrm{Bohr}$, $a^{(1)}=1.0 \mathrm{Bohr}, W_{\infty}^{(1)}=0$ Hartree; $W_{0}^{(2)}=0.1$ Hartree, $R_{0}^{(2)}=2.5 \mathrm{Bohr}, a^{(2)}=1.0 \mathrm{Bohr}, W_{\infty}^{(2)}=0.01$ Hartree. Bottom panel: $W_{x}^{(i)}(R)=W_{0}^{(i)}\left[1-e^{-\left(R-R_{0}^{(i)}\right) / a^{(i)}}\right]^{2}+W_{\infty}^{(i)}, i=1,2$; where $W_{0}^{(1)}=0.1$ Hartree, $R_{0}^{(1)}=5.0$ Bohr, $a^{(1)}=2.5$ Bohr, $W_{\infty}^{(1)}=0$ Hartree; $W_{0}^{(2)}=0.1$ Hartree, $R_{0}^{(2)}=5.01 \mathrm{Bohr}, a^{(2)}=2.53 \mathrm{Bohr}, W_{\infty}^{(2)}=0.01$ Hartree. Right column - The absolute value of $\rho(t)$, the normalized electronic coherence resulting from coherent and incoherent light excitation for the three different PES of the left column. The results for the incoherent light were averaged over a set of 10 random-jump realizations.

values and much faster decoherence rates when the excitation is conducted with pulsed incoherent light than with excitation with coherent pulses of the same center frequency and spectral bandwidth. Thus, even in the absence of external environmental effects, the random character of the incoherent light enhances decoherence. This adds support to the conclusion [10, 12, 17, that longlived electronic coherences observed in biological systems are a result of the use of coherent ultrashort pulses for molecular excitation in these experiments and that such coherences should not be expected in natural processes induced by solar radiation. One would anticipate that the differences noted here between coherent and incoherent excitation would be observable in two dimensional photon echo experiments performed with coherent vs. incoherent light [18].

\section{Acknowledgement}

ACH thanks P. Xiang and E. A. Shapiro for invaluable discussions on the numerical aspects of this work. The 
work of MS was supported by the Natural Sciences and Engineering Research Council of Canada, and that of PB by the US Air Force Office of Scientific Research under contract number FA9550-10-1-0260

[1] G. S. Engel, T. R. Calhoun, E. L. Read, T.-K. Ahn, T. Mančal, Y.-C. Cheng, R. E. Blankenship and G. R. Fleming, Nature, 2007, 446, 782-786.

[2] H. Lee, Y.-C. Cheng and G. R. Fleming, Science, 2007, 316, 1462-1465.

[3] I. P. Mercer, Y. C. El-Taha, N. Kajumba, J. P. Marangos, J. W. G. Tisch, M. Gabrielsen, R. J. Cogdell, E. Springate and E. Turcu, Phys. Rev. Lett., 2009, 102, 057402.

[4] E. Collini, C. Y. Wong, K. E. Wilk, P. M. G. Curmi, P. Brumer and G. D. Scholes, Nature, 2010, 463, 644-647.

[5] G. Panitchayangkoon, D. Hayes, K. A. Fransted, J. R. Carama, E. Harel, J. Wen, R. E. Blankenship and G. S. Engel, Proc. Natl. Acad. Sci. USA, 2010, 107, 1276612770 .
[6] L. A. Pachon and P. Brumer, Phys. Chem. Chem. Phys.

[7] T. Brixner, T. Mančal, I. V. Stiopkin and G. R. Fleming, J. Chem. Phys., 2004, 121, 4221-4236.

[8] X.-P. Jiang and P. Brumer, J. Chem. Phys., 1991, 94, 5833.

[9] H. Hoki and P. Brumer, Procedia Chem., 2011, 3, 122.

[10] P. Brumer and M. Shapiro, Proc. Natl. Acad. Sci. USA (in press).

[11] T. Mančal and L. Valkunas, New J. Phys., 2010, 12, 065044.

[12] L. A. Pachon and P. Brumer, Phys. Rev. A (submitted) arXiv:1210.6374

[13] A. A. Mihajlov, Lj. M. Ignjatović, N. M. Sakan, and M. S. Dimitrijević, Astronomy and Astrophysics, 469, 2, 749, (2007)

[14] V. Engel and H. Metiu, J. Chem. Phys., 1989, 90, 6116.

[15] M. Shapiro, J. Phys. Chem., 1993, 97, 7396.

[16] I. Franco and P. Brumer, J. Chem. Phys., 2012 136, 144501.

[17] I. Kassal, J. Yuen-Zhou and S. Rahimi-Keshari, arXiv:1210.5022

[18] D.B. Turner, D.J. Howey, E.J. Sutor, R.A. Hensrickson, M.W. Gealy and D. J. Ulness, ArXiv 1210.6665 